\title{
Suggestion of a design method for horizontal spiral coil type ground heat exchangers
}

\author{
Min-Jun Kim ${ }^{1}$, Seung-Rae Lee ${ }^{1}$, Seok Yoon ${ }^{2}$, Jun-Seo Jeon ${ }^{1}$ and Min-Seop Kim ${ }^{1}$ \\ ${ }^{1}$ Department of Civil and Environmental Engineering, KAIST, Daejeon, 305-701, \\ Republic of Korea \\ ${ }^{2}$ Radioactive Waste Disposal Division, KAERI, Daejeon, 34057, Republic of Korea
}

Phone/Fax number:+82 42350 3657, e-mail: kmjda1@kaist.ac.kr, srlee@kaist.ac.kr (corresponding author), syoon@kaeri.re.kr, santachi@kaist.ac.kr, gogyu@kaist.ac.kr, hellopdlms@kaist.ac.kr

\begin{abstract}
The need of geothermal energy is constantly increasing for economical and environmental benefits. Horizontal ground heat exchangers (GHEs) can reduce the installation cost and they can also compromise between increasing in efficiency and costs. There are many kinds of GHEs, and it is known that slinky and spiral coil type GHEs show high thermal performance in the horizontal system. However, there is no adequate guideline to design the spiral coil type GHEs even though there is a design program for the slinky type GHEs. Therefore, this paper presents a design module for the horizontal spiral coil type GHEs. Dried Joomunjin standard sand was filled in a steel box including a horizontal spiral coil type GHE of which the size was $5 \mathrm{~m} \times 1 \mathrm{~m} \mathrm{x}$ $1 \mathrm{~m}$ and thermal response tests (TRTs) were conducted for 30 hours to evaluate heat exchange rates. Based on the experimental results, a modified design module, that includes a scheme to properly evaluate design parameters such as ground thermal resistance and borehole thermal resistance used in the Kavanaugh theory for designing a spiral coil type GHE, was suggested.
\end{abstract}

\section{Key words}

Horizontal ground heat exchanger, spiral coil, ground thermal resistance, design module

\section{Introduction}

The need for renewable energy sources is constantly increasing with the advent of global warming and depletion of fossil fuel. Among a variety of renewable energies, geothermal energy has great potential to provide directly usable energy, especially in connection with ground sources. The ground-source heat pump (GSHP) system uses a relatively constant ground temperature to emit heat in summer and to absorb heat during winter for obtaining heating and cooling energy. The GSHP systems can be categorized into open or closed systems. The open systems exchange heat to/from aquifer water, which the closed systems exchange heat to/from the ground by circulating fluid in heat exchange pipes. The closed systems can be divided into vertical and horizontal types.
The vertical system uses vertically installed ground heat exchangers (GHEs) in which circulating fluid flows under the ground to a depth of about 150 200m. This system requires high initial construction costs as a borehole of up to tens or hundreds of meters has to be installed. The horizontal system can be used as an alternative to the vertical system. Since GHEs in the horizontal system are installed at shallow depths of $1 \sim 3 \mathrm{~m}$ parallel to the ground surface, it does not need boring costs which are more expensive than excavation cost in the installation process of the horizontal system. Therefore, the use of horizontal GHEs can reduce the installation cost and minimize the compromise between increase in efficiency and cost [1]. Meanwhile, among various types of horizontal GHEs, slinky and spiral coil type GHEs can reduce horizontal trench length by arranging the pipe as a ring shape instead of a straight line that is used in the horizontal line configuration [2]. Also, Congedo et al. suggested the thermal superiority of spiral coil type GHE in comparison with line and slinky types GHEs [3]. However, while a slinky type GHE can be designed using a ground loop design (GLD) software, which is the only horizontal heat exchanger design program [4], there is no other program or method to accurately design the horizontal spiral coil type GHEs and researches on the horizontal spiral coil type GHEs are insufficient.

Thus, in this study, a design method for the horizontal spiral coil type GHE was suggested based on the previous theory with a reliable analytical solution. Furthermore, the suggested design module was verified by conducting a parametric study using the GLD and a thermal response test (TRT) was conducted to demonstrate the reliability of the analytical solution.

\section{Theoretical Background}

\subsection{Kavanaugh Theory}


The steady state equation of heat transfer from a cylinder in the ground can be transformed to represent the variable heat rate of a GHE by using a series of constant heat rate pulses. The thermal resistance of the ground per unit length is then calculated as a function of time, which corresponds to the time in which a particular heat pulse occurs [5]. Kavanaugh and Rafferty (1997) applied three different pulses of heat: long-term heat imbalances, average monthly heat rates during the design month, and maximum heat rates for a short-term period during the design day [6].

The suggested design length of a borehole for heating and cooling is expressed by equation (1).

$$
L_{H, C}=\frac{q_{a} R_{g a}+q_{l h, l c}\left(\frac{C O P \pm 1}{C O P}\right) \times\left(R_{b}+P L F \cdot R_{g m}+R_{g d} F_{s c}\right)}{T_{g}-\frac{T_{i n}+T_{o u t}}{2}-T_{p}}
$$

Here $\boldsymbol{q}_{\boldsymbol{a}}$ denotes the net annual average heat transfer to the ground, and $\boldsymbol{q}_{\boldsymbol{l h}, \boldsymbol{l} \boldsymbol{c}}$ is the building peak loads for heating and cooling, respectively. $\mathbf{C O P}$ represents a coefficient of performance of a heat pump, $\boldsymbol{P L F}$ is the part-load factor during design month, and $\mathbf{F}_{\mathbf{s c}}$ is the short-circuit heat loss factor. $\mathbf{R}_{\mathbf{b}}$ is the borehole thermal resistance, and $\mathbf{R}_{\mathbf{g a}}, \mathbf{R}_{\mathbf{g m}}$ and $\mathbf{R}_{\mathbf{g d}}$ represent effective thermal resistances for three thermal pulses: an annual pulse, a monthly pulse, and a daily pulse, respectively. $\mathbf{T}_{\mathbf{g}}$ is the undisturbed ground temperature, $\mathbf{T}_{\mathbf{i n}}$ and $\mathbf{T}_{\text {out }}$ are the fluid temperatures at the inlet and outlet, and thus, $\left(\mathbf{T}_{\mathbf{i n}}+\mathbf{T}_{\text {out }}\right) / \mathbf{2}$ represents the arithmetic mean fluid temperature in the pipes. Finally, $\mathbf{T}_{\mathbf{p}}$ is related to the long-term thermal interference effect among the group boreholes and acts as a temperature penalty [7].

\subsection{Heat transfer of ground heat exchanger}

The heat transfer from the GHE to the ground occurs mainly by conduction, even though the heat transfer behaviour between the GHE and the surrounding ground consists of a complicated mechanism [8]. Then, the governing equation for the conduction can be represented by

$$
-\frac{d}{d i}\left(\lambda \frac{d T}{d i}\right)+\rho c \frac{d T}{d t}+q_{i}=0 \quad(i=x, y, z)
$$

Here, $\mathrm{T}$ is the ground temperature, $\lambda$ is the ground thermal conductivity, $\rho$ is the density, $\mathrm{c}$ is the specific heat capacity, and $q_{i}$ is the internal heat generation. One of the reliable methods of solving the heat transfer equation is to use the heat source models such as the infinite line source, cylindrical source model, and so on.

\section{Design Module}

\subsection{Description}

In the design of a total required pipe length in a vertical and horizontal GHE, there are two major differences. First, while the installation depth of a vertical GHE is $150 \sim 200 \mathrm{~m}$ deep under the ground, a horizontal GHE is installed in a trench approximately $1.5 \sim 3 \mathrm{~m}$ deep. Accordingly, the ground temperature at the installation depth of a horizontal GHE can be significantly affected by the ambient conditions. Therefore, the ground temperature should be accurately calculated with regard to the installation depth of a horizontal GHE. Second, in the vertical system, the required borehole length is obtained by considering a borehole which contains the grouts and vertical GHE as a heat source/sink in the ground. On the other hand, there is no borehole in the horizontal system.

Thus, in the present study, a design module for a horizontal GHE was suggested by transforming the Kavanaugh theory for a borehole of the vertical GHE to the one for a trench of the horizontal GHE as in (3).

$$
L_{H, C}=\frac{q_{a} R_{g a}+q_{l h, l c}\left(\frac{C O P \pm 1}{C O P}\right) \times\left(R_{p}+F_{H} R_{g m}+R_{g d} F_{s c}\right)}{T_{L, H}-\frac{T_{i n}+T_{o u t}}{2}-T_{p}}
$$

Here, $\mathbf{R}_{\mathbf{p}}$ is the pipe thermal resistance and $\mathbf{T}_{\mathbf{L}, \mathbf{H}}$ are the minimum and maximum ground temperatures at the installation depth of a horizontal GHE, respectively, instead of the undisturbed ground temperature. Moreover, in determining $\boldsymbol{F}_{\boldsymbol{H}}$ and $\boldsymbol{F}_{\boldsymbol{S} \boldsymbol{C}}$, we should consider the different condition of horizontal GHE.

\subsection{Verification}

In order to verify the reliability for use of the suggested design module, parametric studies were conducted using GLD. The GLD can calculate a design trench length of a horizontal GHE by entering the building load, heat pump, soil, fluid, and GHE configuration data. To obtain the input data for using GLD, a certain office of which the size is $10 \mathrm{~m} \times 10 \mathrm{~m}$ was selected as a target building and the building loads were obtained by using a commercial program called Design builder [9] (Fig. 1).

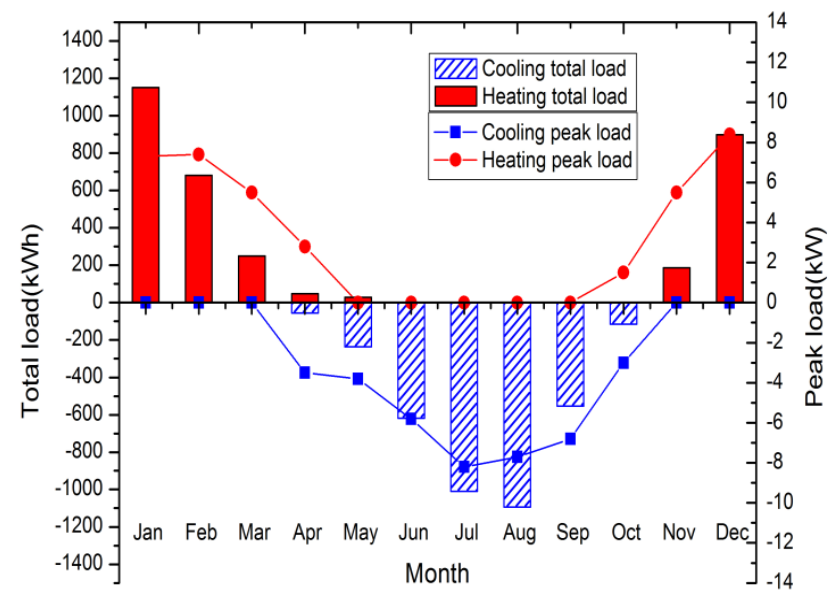

Fig. 1. Monthly building loads

Table 1 presents detailed information for the horizontal GHE design in GLD. The specification of a heat pump was selected automatically in GLD according to the building loads and other input data were arbitrarily set 
considering a common installation depth of a horizontal GHE and a line type configuration.

With the input data, the horizontal GHE lengths for heating were obtained by using GLD and the suggested design module, respectively for the specific building as shown in Table 2.

Table 1. Input data and Specification

\begin{tabular}{c|c|c}
\hline & Input data & Value \\
\hline \multirow{4}{*}{$\begin{array}{c}\text { Build- } \\
\text { ing }\end{array}$} & Total ground area $\left(\boldsymbol{m}^{\mathbf{2}}\right)$ & 100 \\
\cline { 2 - 3 } & Total heating load $(\mathrm{kWh})$ & 3247 \\
\cline { 2 - 3 } & Total cooling load $(\mathrm{kWh})$ & 3682 \\
\cline { 2 - 3 } & Peak heating load $(\mathrm{kW})$ & 8.4 \\
\hline \multirow{2}{*}{$\begin{array}{c}\text { Heat } \\
\text { pump }\end{array}$} & Peak cooling load $(\mathrm{kW})$ & 8.2 \\
\cline { 2 - 3 } & COP $($ Heating/Cooling $)$ & $3.9 / 3.7$ \\
\hline \multirow{4}{*}{ Soil } & Ground temperature $\left({ }^{\circ} \mathrm{C}\right)$ & $4.4 / 1.1$ \\
\cline { 2 - 3 } & Thermal conductivity $(\mathrm{W} / \mathrm{mK})$ & 16 \\
\cline { 2 - 3 } & Thermal diffusivity $\left(\boldsymbol{m}^{2} / \boldsymbol{d}\right)$ & 0.116 \\
\hline Pipe & Size $($ outer/inner diameter $)(\mathrm{mm})$ & $26.7 / 25$ \\
\hline \multirow{3}{*}{ GHE } & Installation depth $(\mathrm{m})$ & 1.6 \\
\cline { 2 - 3 } & Circulating fluid & Water \\
\cline { 2 - 3 } & Configuration & 1 x1, Line-type \\
\hline
\end{tabular}

Table 2. Horizontal GHE length

\begin{tabular}{c|c|c}
\hline & GLD & Suggested design module \\
\hline $\begin{array}{c}\text { Total pipe } \\
\text { length }(\mathrm{m})\end{array}$ & 352.5 & 357.2 \\
\hline
\end{tabular}

To compare the effects of soil properties and building loads on the design length of each design method, parametric studies were conducted. According to Kim et al.[2], the soil thermal conductivity is a critical factor for designing a horizontal GHE, and thus it was chosen as a parametric study factor on behalf of soil properties. Also, a peak load was selected as a factor of representing the building loads and heat pump specification. The results of parametric studies were shown in Fig. 2 and Fig. 3.

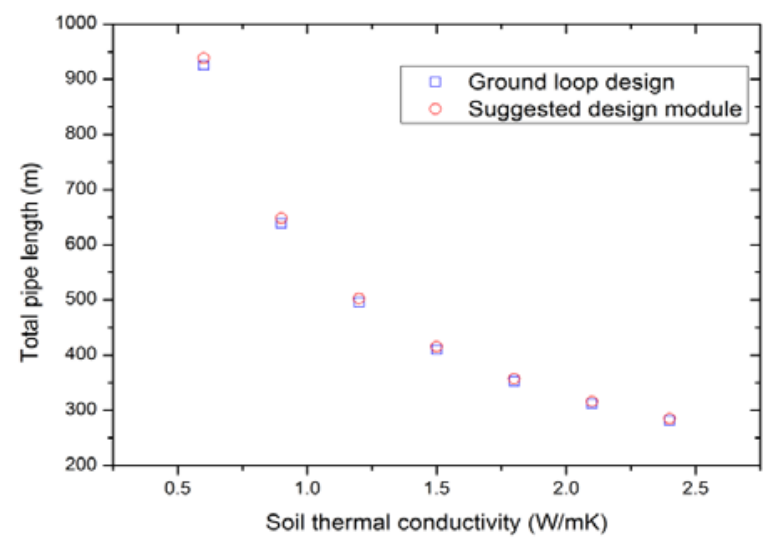

Fig. 2. Comparison of pipe lengths according to the soil thermal conductivity variation

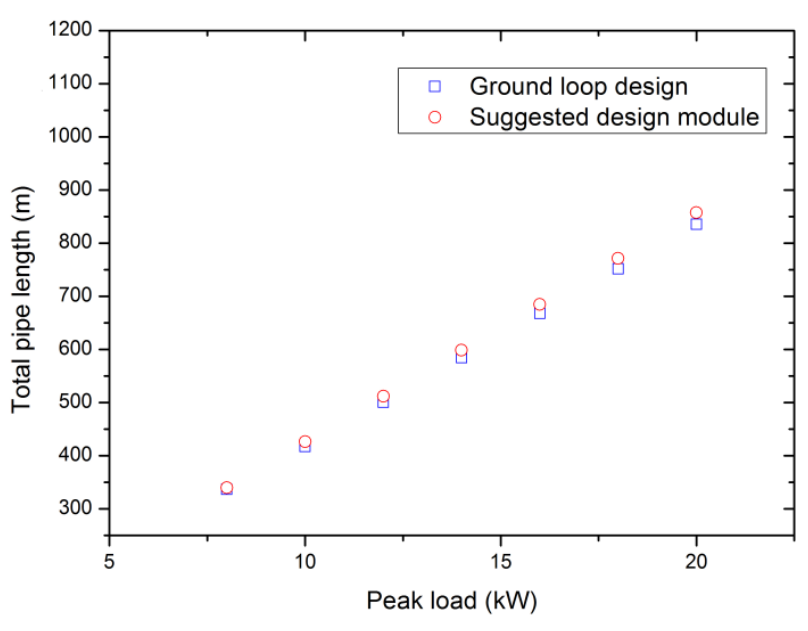

Fig. 3. Comparison of pipe lengths according to the peak load variation

As shown in Fig. 2 and Fig. 3, the total pipe lengths calculated by both methods were almost identical according to both the soil thermal conductivity and peak load variations. Thus, it can be concluded that the suggested design module can predict the design horizontal GHE pipe length with regard to the line configuration as well.

\section{Ground Thermal Resistance}

\subsection{Description}

The ground thermal resistance in the design of HGHE represents the resistance to heat transfer between the outer wall of the buried pipe and the design heating or cooling soil temperature, and it depends on the diameter, number, and configuration of the pipe(s) in the trenches [8]. Thus, in order to develop a design method of a spiralcoil type GHE for the previously suggested design module, ground thermal resistances $\left(\boldsymbol{R}_{\boldsymbol{g a}}, \boldsymbol{R}_{\boldsymbol{g m}}, \boldsymbol{R}_{\boldsymbol{g d}}\right)$ should appropriately include the information of the configuration of a spiral coil type GHE. For the calculation of ground thermal resistances of a spiral coil type GHE, a spiral coil source (SCS) model was additionally built in the suggested design module.

The SCS model was developed by Park (2012) and it is an analytical solution of spiral coil type GHE by considering the three dimensional shape effects and the radial dimension effect of spiral coil type GHEs using Green's function. For modelling the heat transfer from the buried spiral coil type GHE in the analytical solution, the following assumptions were made:

(1) The ground is assumed to be a homogeneous infinite medium. The thermal properties of the ground do not change with the variation of temperature.

(2) The spiral coil heat source is assumed to take a spiral coil form without a vertical outlet pipe. The spiral coil heat source is buried in the ground from the ground surface to a certain depth, h (Fig. 4). The mass, heat capacity and thickness of the heat source are neglected. The heating rate per length of pipe, $\boldsymbol{q}_{\boldsymbol{l}}$, is constant from the starting instant, $\mathrm{t}=0$. 


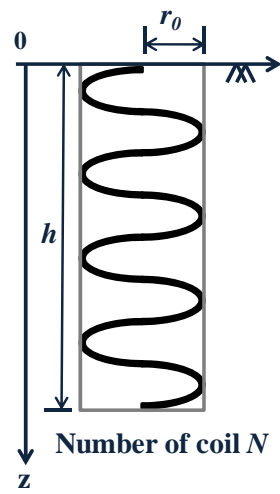

Fig. 4. Schematic diagram of spiral coil source

(3) The medium has a uniform initial temperature, $\boldsymbol{t}_{\mathbf{0}}$.

(4) The ground surface as the boundary of the medium, $\mathrm{z}=0$, maintains a constant temperature.

The solution derived using this model can be expressed as in (4).

$$
\begin{gathered}
\theta(u, t)=\frac{Q / L}{4 \pi \lambda} \int_{0}^{h} \frac{\operatorname{erfc}\left(A_{-}\left(u, z^{\prime}\right) / 2 \sqrt{\alpha t}\right)}{A_{-}\left(u, z^{\prime}\right)}-\frac{\operatorname{erfc}\left(A_{+}\left(u, z^{\prime}\right) / 2 \sqrt{\alpha t}\right)}{A_{+}\left(u, z^{\prime}\right)} d z^{\prime} \\
\text { with } A_{ \pm}\left(u, z^{\prime}\right)=\sqrt{F\left(x, y, z^{\prime}\right)+\left(z \pm z^{\prime}\right)^{2}}
\end{gathered}
$$

The model used an error function to overcome the computational problems of previous models such as solid cylindrical source model and ring coil source model which are used in the analysis of spiral coil source. Park et al.(2012) evaluated the accuracy of SCS model by experimental and numerical methods and they demonstrated that the suggested model is more accurate than the previous models. Thus, the SCS model was selected to be implemented in the design module for the spiral coil GHE and the reliability was verified by experiments.

\subsection{Verification}

A TRT was conducted with the experimental setup including an electric heater with a circulating pump, a water tank, and a spiral-shaped polybutylene (PB) heat exchanger to validate the use of SCS model in the design module. In the experiment, a $5 \mathrm{~m} \times 1 \mathrm{~m} \times 1 \mathrm{~m}$ steel box filled with dry Joomunjin standard sand (Table 3) was used to reflect the surrounding ground, while the TRT equipment was installed to supply the circulating water and constant power to the inside of the heat exchanger buried in the ground (Fig. 5).

Table 3. Properties of standard Joomunjin sand

\begin{tabular}{c|c}
\hline Properties & Value \\
\hline Uniformity Coefficient, $\mathrm{c}_{\mathrm{u}}$ & 2.06 \\
\hline Curvature Coefficient, $\mathrm{c}_{\mathrm{c}}$ & 1.05 \\
\hline
\end{tabular}

\begin{tabular}{c|c}
\hline Specific Gravity, $\mathrm{G}_{\mathrm{s}}$ & 2.65 \\
\hline Maximum Dry Density, $\gamma_{\mathrm{dmax}}\left(\mathrm{kN} / \mathrm{m}^{3}\right)$ & 16.17 \\
\hline Minimum Dry Density, $\gamma_{\mathrm{dmin}}\left(\mathrm{kN} / \mathrm{m}^{3}\right)$ & 13.49 \\
\hline Water Content, $\mathrm{w}(\%)$ & 0 \\
\hline
\end{tabular}

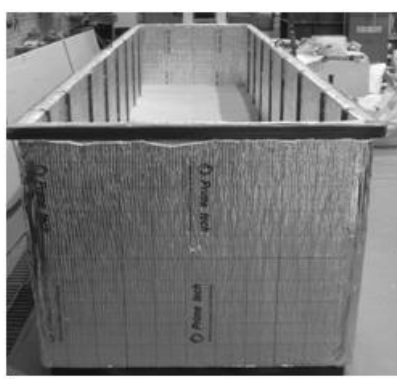

(a) Steel box setting

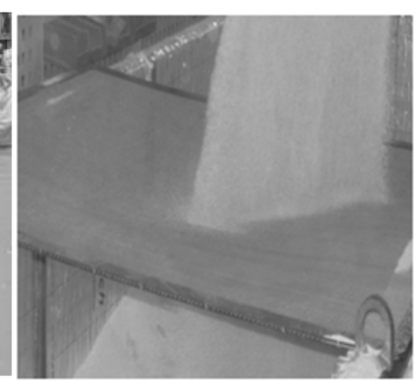

(b) Composition of sample

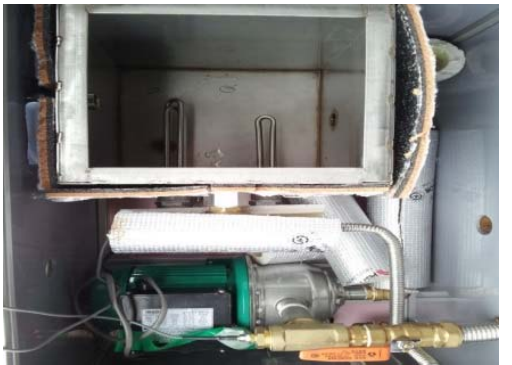

(c) TRT equipment

Fig. 5. Process of TRT

The outer and inner diameters of the PB pipe were $25 \mathrm{~mm}$ and $20 \mathrm{~mm}$, respectively. In order to measure the temperature change in the surrounding ground, resistance temperature detector (RTD) sensors were installed at $10 \mathrm{~cm}$ away from the edge of GHE (Fig. 6). The initial temperature of Joomunjin sand was 17.46 degree and the average flow rate of the circulating water was $4.73 \mathrm{lpm}$.

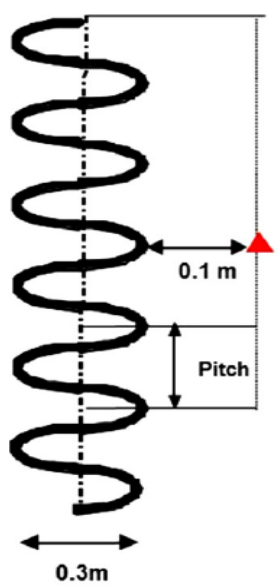

Fig. 6. Diagram of hotizontal GHE and sensors

The experiment was conducted for 30 hours continuously to measure the ground temperature using the temperature sensors. The ground temperature variations of the experiment and analytical solution are shown in Fig. 7. 


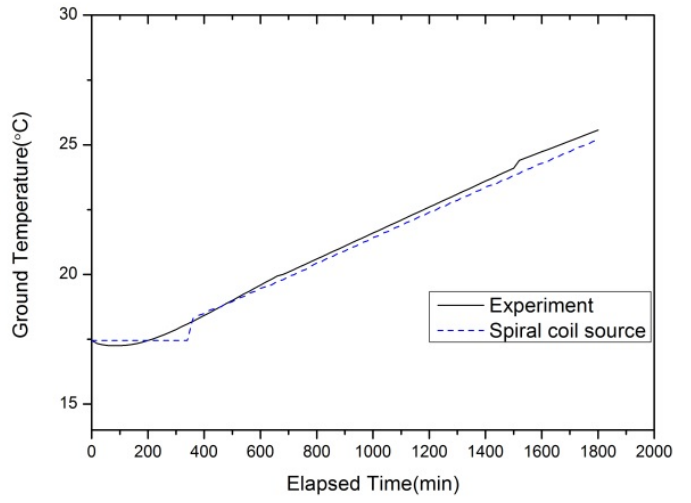

Fig. 7. Ground temperature variations obtained from TRT and spiral coil source model

In the experiment, the ground temperature continuously increased because of the heat conduction from a heat source induced by the horizontal pipe. The results show that the SCS model result matches well with the actual experimental result.

For comparison of the results, at first, the ground thermal resistances were obtained using the injected heat and the measured ground temperature changes after a certain elapsed time in the experiment. Then, using the heat source model, a ground thermal resistance was calculated to compare it with one measured from the experiment. The comparison results are summarized in Table 4.

Table 4. Comparison of ground thermal resistances

\begin{tabular}{c|c}
\hline $\begin{array}{c}\text { Determination } \\
\text { Methods }\end{array}$ & Ground Thermal Resistance $(\mathrm{K} \cdot \mathrm{m} / \mathrm{W})$ \\
\hline Experiment & 0.124 \\
\hline $\begin{array}{c}\text { Spiral coil source } \\
\text { model }\end{array}$ & 0.119 \\
\hline
\end{tabular}

Therefore, we concluded that the model can accurately estimate the ground thermal resistance of a horizontal spiral coil type GHE and used it in the suggested design module for the design of a horizontal spiral coil type GHE.

\section{Conclusion}

In the present study, a design module for a horizontal GHE was suggested. In order to verify the applicability of the module, parametric studies and a TRT were conducted using GLD and a $5 \mathrm{~m} \times 1 \mathrm{~m} \times 1 \mathrm{~m}$ steel box filled with Joomunjin standard sand. The results showed that the suggested design module corresponds well with GLD results for a line type GHE and the SCS model implemented in the design module accurately predicts the actual experimental results for a spiral coil type GHE.

Therefore, it can be concluded that it is beneficial to use the suggested design module to design the horizontal spiral coil type GHE, because the design method for the type does not exist currently. However, additional researches are still required with regard to the application of the suggested module to the versatile design program.

\section{Acknowledgment}

This research was supported by a Regional Development Research Program (15RDRP-B076564-02) of the Ministry of Land, Infrastructure and Transport of the Korean Government and by a basic research project (2013R1A2A2A01067898) of the National Research Foundation of Korea.

\section{References}

[1] H. Demir, A. Koyun and G. Temir, "Heat transfer of horizontal parallel pipe ground heat exchanger and experimental verification", Appl. Therm. Eng, 2009, Vol. 29, pp. 224-233.

[2] M. J. Kim, S. R. Lee, S. Yoon and G. H. Go "Thermal performance evaluation and parametric study of a horizontal ground heat exchanger", Geothermics, 2015, accepted.

[3] P.M. Congdeo, G. Colangelo, G. Starace, CFD simulation of horizontal groundheat exchangers: a comparison among different configurations, Appl. Therm.Eng., 2012, 33-34 pp. 24-32.

[4] Gaia Geothermal Group Loop Design Software, GLD2012.

[5] S. Kavanaugh and K. Rafferty "Ground-source heat pumps : design of geothermal systems for commercial and institutional buildings", ASHRAE, USA, 1997.

[6] L. Lamarche, G. Dupre and S. Kail, "A New Design Approach for Ground Source Heat Pumps Based on Hourly Load Simulations", Geothermics, 2010, 39, pp. 187-200.

[7] S. Yoon, "Suggestion of Design Parameters and Evaluation of Thermal Efficiency in Energy Piles by Thermal Performance Tests", Ph.D. Dissertation, Korea Advanced Institute of Science and Technology, Daejeon, Republic of Korea, 2016.

[8] H. Brandl, "Energy foundations and other thermos-active ground structures", Geotechnique, 2006, 56(2),pp. 81-122.

[9] Design Builder Training Manual Ver. 5.2b, Design Builder Software Ltd, UK, 2014. 